\title{
Novel homozygous variant in the TPO gene associated with congenital hypothyroidism and mild-intellectual disability
}

\author{
Amjad Khan (1)', Muhammad Umair (1)', Rania Abdulfattah Sharaf², Muhammad Ismail Khan ${ }^{3}$, Amir Ullah", \\ Safdar Abbas ${ }^{5}$, Nargis Shaheen ${ }^{6}$, Muhammad Bilal ${ }^{5}$ and Farooq Ahamd ${ }^{7}$
}

\begin{abstract}
Congenital hypothyroidism $(\mathrm{CH})$ is one of the most common hereditary disorders affecting neonates worldwide. $\mathrm{CH}$ is a multifactorial complex disorder and can be caused by either environmental factors or genetic factors. We studied one Pakistani family with segregating mutations in $\mathrm{CH}$ inherited in an autosomal recessive manner. Using wholeexome sequencing (WES), we found a novel homozygous missense variant (c.2315A>G; p.Tyr772Cys) in the thyroid peroxidase (TPO) gene. Different bioinformatics prediction tools and Sanger sequencing were performed to verify the identified variant. Our findings highlight the importance of this gene in causing $\mathrm{CH}$ and mild-intellectual disability (ID) in two affected brothers. WES is a convenient and useful tool for the clinical diagnosis of $\mathrm{CH}$ and other associated disorders.
\end{abstract}

\section{Introduction}

Congenital hypothyroidism $(\mathrm{CH})$ is diagnosed as thyroid hormone deficiency in newborn infants with an incidence of 1:2000 to 1:4000 live births worldwide ${ }^{1}$. Approximately $80-85 \%$ of $\mathrm{CH}$ cases are associated with thyroid dysgenesis (TD); either the thyroid gland is absent, reduced in size or versatile ${ }^{2}$. Upon the diagnosis of $\mathrm{CH}$, early $\mathrm{TH}$ administration is a key to avoid severe structural, motor, and neurodevelopmental defects ${ }^{3}$. Molecular investigations assist in definitive diagnosis and precise classification of $\mathrm{CH}$ and might illustrate patientspecific targets for alternative treatment of the disease. Currently, there are a handful of genes known to be

\footnotetext{
Correspondence: Amjad Khan (amjadkhanqau123@hotmail.com)

${ }^{1}$ Medical Genomics Research Department, King Abdullah International Medical Research Center (KAIMRC), King Saud bin Abdulaziz University for Health Science, Ministry of National Guard-Health Affairs (MNGHA), Riyadh, Saudi Arabia

2Department of Speech Language Pathology and Audiology, National Guard Health Affairs, Ministry of National Guard, Riyadh, Saudi Arabia Full list of author information is available at the end of the article These authors contributed equally: Amjad Khan, Muhammad Umair
}

responsible for $\mathrm{CH}$ associated with both primary thyroid dysgenesis and thyroid dyshormonogenesis (TDH) ${ }^{4}$.

In this report, we describe a consanguineous Pakistani family with two affected individuals (IV: 2, IV: 5) with $\mathrm{CH}$ and ID. Signed informed consent for the genetic analysis and publication of data was obtained from the patient's legal guardians. A pedigree was generated (Fig. 1A), and the affected individuals were thoroughly examined by a local endocrinologist and geneticist. Index patient IV: 2 was diagnosed with $\mathrm{CH}$ and mild ID at the age of 26 when he underwent a thorough examination for prolonged jaundice. At the age of 26 years, patient IV: 2 had tall forehead, thick eyebrows, deep-set eyes, strabismus, thick lips, protruding ears, and a prominent goiter. He had already undergone thyroidectomy twice at the age of 11 and 20 years. The goiter size has gradually increased over the last few years. Family history revealed a brother (IV: 5 ) with $\mathrm{CH}$ and mild ID (IQ score 54) diagnosed at the age of 24. Patient IV: 5 had a prominent supraorbital ridge, with mild nasal flaring, no bulbous nasal tip, deep-set eyes, thick upper and lower lip, and pointed chin. He had normal ears and a short forehead. Hypoplastic philtrum, 


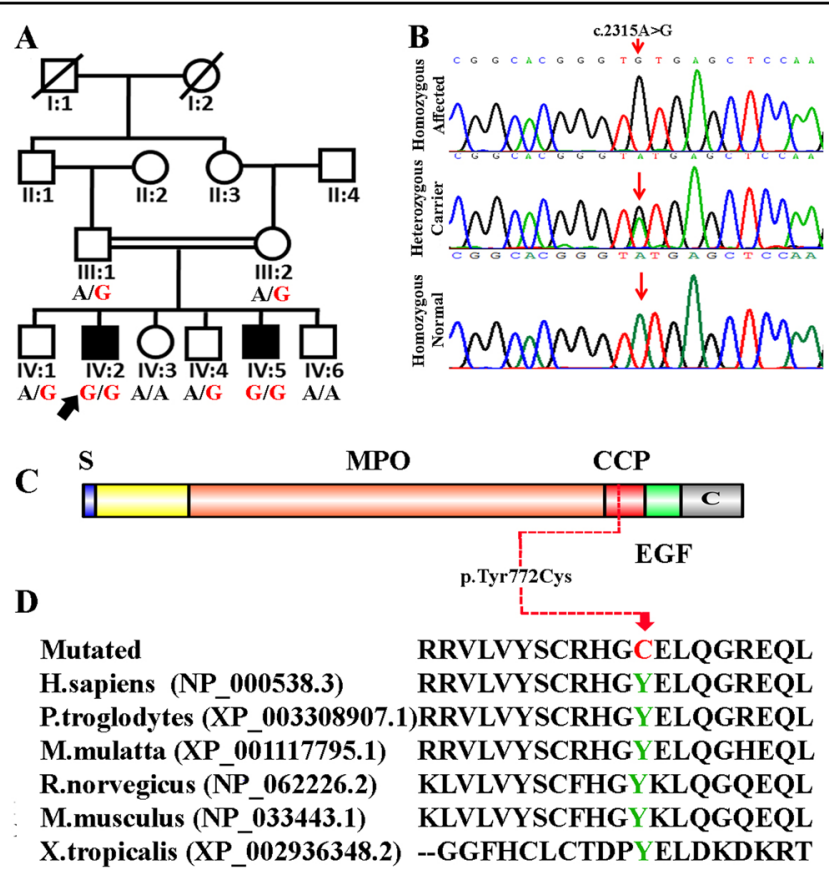

Fig. 1 Genetic and molecular analysis. A A consanguineous pedigree showing two affected members (IV: 2 and IV: 5) in the fourth generation with congenital hypothyroidism. Affected individuals in the pedigree are shown with shaded symbols, and unaffected individuals are indicated with open symbols. Double lines indicate a consanguineous union. B Sequence chromatogram showing the missense TPO variant. The red arrow indicates the site of the identified variant in this study. Affected individuals (IV: 2 and IV: 5) are homozygous, parents (III: 1 and III: 2) and two normal brothers (IV: 1 and IV: 4) are heterozygous, and two siblings (IV: 3 and IV: 6) are homozygous normal for the identified variant. C Schematic representation of TPO protein and its domains; arrow indicates the location of the mutation identified in the present study. D Conservation of p.Tyr 772 across several species.

social and behavioral abnormalities, prominent goiter, ID (IQ: 54), and squint eye were noted. Further endocrine and laboratory workups showed thyroxin (T4) $1.50 \mu \mathrm{g} / \mathrm{dl}$, free $\mathrm{T} 40.4 \mathrm{ng} / \mathrm{dl}$, triiodothyronine (T3) $7.4 \mathrm{ng} / \mathrm{ul}$, thyroid stimulating hormone (TSH) $62 \mu \mathrm{U} / \mathrm{ml}$, thyroglobulin (TG) $5.1 \mathrm{ng} / \mathrm{ml}$, and thyroxine-binding globulin (TBG) $23 \mu \mathrm{g} / \mathrm{ml}$.

For molecular investigation, fresh blood samples were drawn from the affected and normal siblings and parents. Genomic DNA was extracted using the QIAquick DNA Extraction Kit (Qiagen, Hilden, Germany). Whole-exome sequencing (WES) and data analysis were performed as described previously ${ }^{5,6}$. All variants were screened according to the location, frequency, and type of mutation (Supplementary Tables 1 \& 2). We also focused on 21 known genes implicated in $\mathrm{CH}$ (Supplementary Table 3 ) and only found a novel homozygous missense variant (c.2315A $>$ G; p. Tyr772Cys) in the TPO (NM_175719.3; rs1382787497) gene in both affected individuals (IV: 2 and IV: 5), which was confirmed by Sanger sequencing (Fig. 1B). The parents (III: 1 and III: 2 ) and two normal brothers (IV: 1 and IV: 4) were heterozygous, and the two siblings (IV: 3 and IV: 6) were homozygous normal for this variant (Fig. 1B).

Different bioinformatics tools, including Mutation Taster (http://www.mutationtaster.org/), Polyphen-2 (http://genetics.bwh.harvard.edu/pph2), Sorting Intolerant From Tolerant (SIFT, http://www.sift.jcvi.org/), Exome Sequencing Project (ESP, http://evs.gs.washington. edu/EVS/), Protein Variation Effect Analyzer (PROVEAN, http://www.provean.jcvi.org), Human Splicing Finder (HSF, http://www.umd.be/HSF/), Combined Annotation Dependent Depletion (CADD, https://cadd.gs. washington.edu/) and Varsome (https://varsome.com/), were used for functional effect prediction. The variant (c.2315A>G; p.Tyr772Cys) was also analyzed in 200 ethnically matched control individuals and 145 in-house (Pakistani) exomes. Finally, the American College of Medical Genetics and Genomics (ACMG) 2015 criteria and guidelines (PM2, PP3, PP2, PP1, and PP4) were used for the interpretation of variants that were classified as likely pathogenic ${ }^{7}$. The identified variant is located in a highly conserved complement control protein (CCP)-like domain in the TPO gene, which might affect the secondary structure and binding to other important proteins involved in the proper function of TPO (Fig. 1C, D). The TPO gene is located on chromosome $2 \mathrm{p} 25$ and has 17 exons, consisting of $150 \mathrm{~kb}$ of DNA and encodes a 933 amino acid-TPO enzyme.

The crystal structure resolved at $1.99 \AA$ resolution (PDB ID: $3 Z D 2)^{8}$ was used for the wild-type and mutant model 


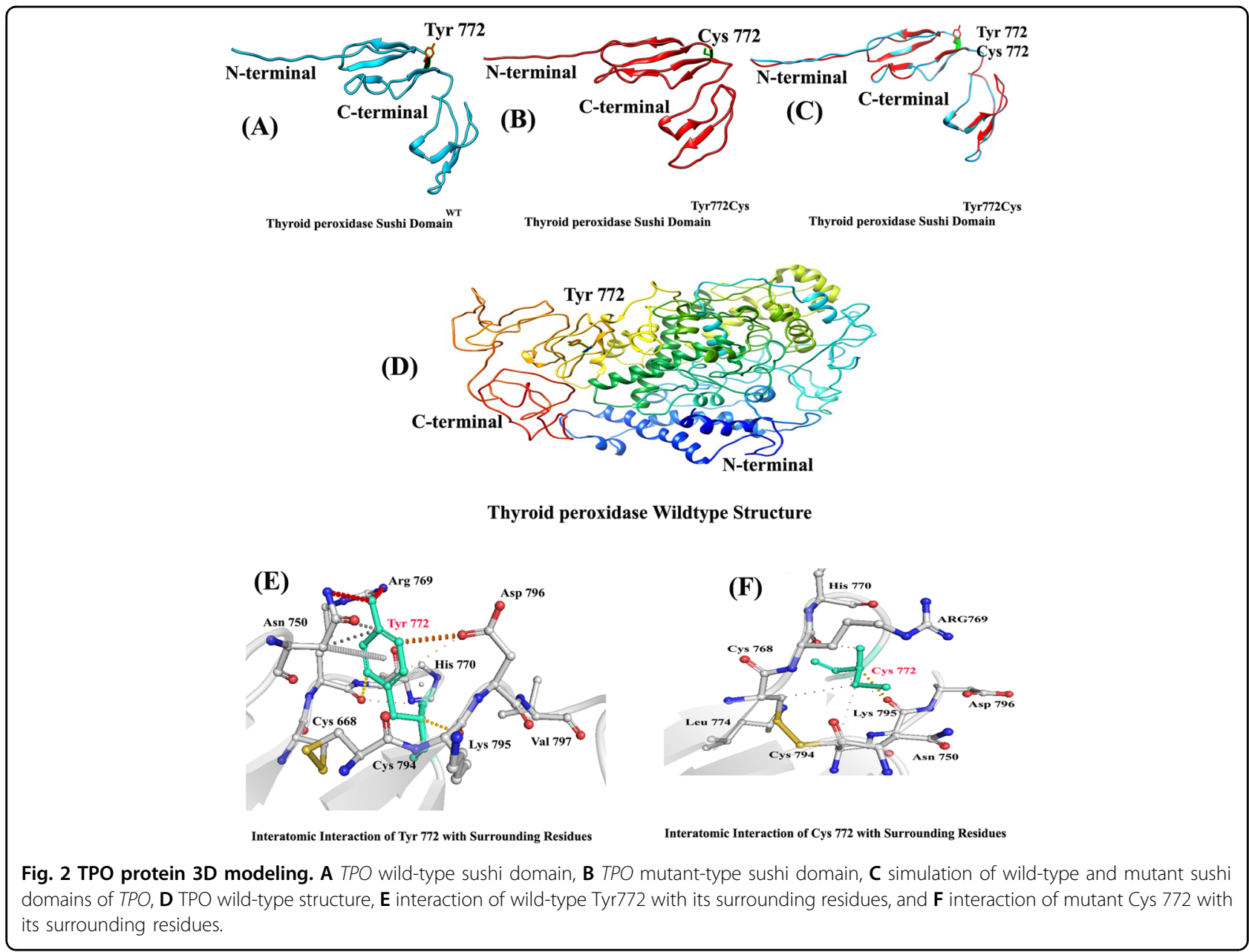

structure and analysis. The amino acid (993 aa) sequence of the TPO-encoding protein was retrieved from the UniProt database with accession number P07202-1 in FASTA format. Structure visualization, measurement of distance, and mutagenesis analysis were performed with different bioinformatics software programs as described previously, ${ }^{5,9}$ Our analysis revealed that Tyr772 interacts with Asn750, Cys668, His770, Arg769, Lys795, and Asp 796 (Fig. 2A-F). Tyrosine is an aromatic polar amino acid, but substitution of a smaller cysteine to a larger tyrosine disrupts its interaction with surrounding amino acid residues, and these new interactions in turn might potentially disrupt both protein secondary structure and function. Using DUET, ENCoM, SDM, and MCSM, we predicted that the Tyr772Cys mutation would cause a $-0.585,-0.502,-1.28$, and $-0.54 \mathrm{kcal} / \mathrm{mole}$ change in the $\Delta \Delta G$, respectively, indicating that the mutation would greatly destabilize the protein structure and hence disrupt function.

To date, 161 mutations (missense, nonsense, splice site and frameshift) associated with $\mathrm{CH}$ phenotypes have been described in the human gene mutation database (HGMD; http://www.hgmd.cf.ac.uk/ac/index.php) (Supplementary Table 4). Cangül et al. ${ }^{10}$ studied a consanguineous Turkish family with a homozygous nonsense mutation (c.1618C > T; p. p.R540X) in the TPO gene, leading to $\mathrm{CH}^{11}$. Another study on two Amish families by Pannain et al. identified homozygous missense mutations (c.2395G >A; p.Glu799Lys, and c.1943G >A; p.Arg648Gln) in the TPO gene with $\mathrm{CH}^{12}$. Fu et al. ${ }^{1}$ examined the TPO mutation spectrum and prevalence among 192 patients with $\mathrm{CH}$ in the Guangxi Zhuang Autonomous Region of China and described the genotypic-phenotypic relationship with TPO mutation. A literature study suggests that mutations in the TPO gene are one of the most common causes of ID and $\mathrm{CH}$ in the Pakistani population ${ }^{13}$. Families with segregating mutations in these genes should be counseled either for genetic or blood-based screening, and preventive therapies should be applied. Recent developments in DNA sequencing technology such as NGS can improve the diagnostic toolset that might help to identify novel causes for $\mathrm{CH}$ and related disorders. In general, such data will be helpful in diagnosing and predicting $\mathrm{CH}$ /goitrous hypothyroidism, and inborn screening of TPO gene 
mutations will be valuable for the identification of affected newborns or gene carriers in families.

\section{HGV database}

The relevant data from this Data Report are hosted at the Human Genome Variation Database at https://doi.org/10.6084/m9.figshare.hgv.2942

\section{Acknowledgements}

We thank all the individuals for their participation in this study.

\section{Author details}

${ }^{1}$ Medical Genomics Research Department, King Abdullah International Medical Research Center (KAIMRC), King Saud bin Abdulaziz University for Health Science, Ministry of National Guard-Health Affairs (MNGHA), Riyadh, Saudi Arabia. ${ }^{2}$ Department of Speech Language Pathology and Audiology, National Guard Health Affairs, Ministry of National Guard, Riyadh, Saudi Arabia. ${ }^{3}$ Department of Zoology, Islamia College University, Peshawar, Pakistan. ${ }^{4}$ Nephrology and Dialysis Unit, District Head Quarter (DHQ) Teaching Hospital, Bannu 2800, Pakistan. ${ }^{5}$ Department of Biochemistry, Faculty of Biological Sciences, Quaid-i-Azam University, Islamabad, Pakistan. ${ }^{6}$ Department of Zoology, Faculty of Biological Sciences, Quaid-i-Azam University, Islamabad, Pakistan. ${ }^{7}$ Department of Chemistry, Swabi University, KPK, Pakistan

\section{Author contributions}

A.K., M.U., and M.I.K. were involved in the planning of the experiments. A.U. and N.S. extracted DNA from the proband and her healthy parents' samples, and R.A.S. performed polymerase chain reaction. M.B. and F.A. performed the WES experiment. A.K. and M.U. analyzed the obtained WES results, performed bioinformatics analysis, and supervised the findings of this work. A.K. wrote the manuscript with consultation and support from M.U. All authors read and approved the final manuscript.

\section{Data availability}

The datasets supporting the conclusions of this article are included within the article and its additional file.

\section{Conflict of interest}

The authors declare that they have no conflict of interest.

\section{Consent for publication}

Informed written consent for publication of the participants' and clinical details were obtained from their parents or legal guardians or the participants who were over the age of 18 .

\section{Publisher's note}

Springer Nature remains neutral with regard to jurisdictional claims in published maps and institutional affiliations.
Supplementary information is available for this paper at https://doi.org/ 10.1038/s41439-020-00129-3.

Received: 10 June 2020 Revised: 13 August 2020 Accepted: 18 August 2020.

Published online: 27 November 2020

\section{References}

1. Fu, C. et al. Mutation screening of the TPO gene in a cohort of 192 Chinese patients with congenital hypothyroidism. BMJ Open 6, e010719 (2016).

2. Macchia, P. E. et al. PAX8 mutations associated with congenital hypothyroidism caused by thyroid dysgenesis. Nat. Genet. 19, 83-86 (1998).

3. Medeiros-Neto G., Knobel M., DeGroot L. J. in Genetics in Endocrinology (ed Baxter J. D.) 375-402 (Williams \& Wilkins, Philadelphia, Lippincott, 2002).

4. Castanet, M. et al. AFDPHE (Association Francaise pour le Depistage et la Prevention des Handicaps de l'Enfant). Nineteen years of national screening for congenital hypothyroidism: familial cases with thyroid dysgenesis suggest the involvement of genetic factors. J. Clin. Endocrinol. Metab. 86, 2009-2014 (2001).

5. Khan, A. et al. Homozygous missense variant in the TTN gene causing autosomal recessive limb-girdle muscular dystrophy type 10. BMC Med. Genet. 20, 166 (2019).

6. Wang, R., Khan, A., Han, S. \& Zhang, X. Molecular analysis of 23 Pakistani families with autosomal recessive primary microcephaly using targeted nextgeneration sequencing. J. Hum. Genet. 62(Feb), 299-304 (2017).

7. Richards, S., ACMG Laboratory Quality Assurance Committee. et al. Standards and guidelines for the interpretation of sequence variants: a joint consensus recommendation of the American College of Medical Genetics and Genomics and the Association for Molecular Pathology. Genet. Med. 17, 405-424 (2015).

8. Goicoechea de Jorge, E. et al. Dimerization of complement factor H-related proteins modulates complement activation in vivo. Proc. Natl Acad. Sci. USA 110, 4685-4690 (2013).

9. Umair, M. et al. Biallelic missense mutation in the ECEL1 underlies distal arthrogryposis type 5 (DA5D). Front. Pediatr. 7, 343 (2019).

10. Cangül, H., Doğan, M. \& Üstek, D. A homozygous nonsense thyroid peroxidase mutation (R540X) consistently causes congenital hypothyroidism in two siblings born to a consanguineous family. J. Clin. Res. Pediatr. Endocrinol. 7(4), 323-328, https://doi.org/10.4274/jcrpe.1920 (2015).

11. Pannain, S. et al. Two different mutations in the thyroid peroxidase gene of a large inbred Amish kindred: power and limits of homozygosity mapping. J. Clin. Endocrinol. Metab. 84, 1061-71 (1999).

12. Rodrigues, $\mathrm{C}$. et al. Mutation screening of the thyroid peroxidase gene in a cohort of 55 Portuguese patients with congenital hypothyroidism. Eur. J. Endocrinol. 152, 193-198 (2005).

13. Mittal, K. et al. Mutations in the genes for thyroglobulin and thyroid peroxidase cause thyroid dyshormonogenesis and autosomal-recessive intellectual disability. J. Hum. Genet. 61, 867-872 (2016). 